\title{
Assessing Organic Chemical Emissions and Workers' Risk of Exposure in a Medical Examination Center Using Solid Phase Microextraction Devices
}

\author{
Wen-Hsi Cheng ${ }^{{ }^{*}}$, Hui-Min $\mathrm{Wu}^{2}$ \\ ${ }^{1}$ Department of Occupational Safety and Hygiene, Fooyin University, Kaohsiung 83102, Taiwan \\ ${ }^{2}$ Center of Occupational Safety and Hygiene, Fooyin University Hospital, Pingtung 92847, Taiwan
}

\begin{abstract}
Needle trap samplers (NTS), which are environmentally friendly solid phase microextraction sampling devices, were used to obtain air samples at a medical examination center in a teaching hospital to determine the concentration of airborne xylene. The standard active sampling method, Method 1501, was simultaneously used to evaluate the exposure of workers to xylene. The concentrations of xylene were much lower than the legal 100-ppm time-weighted average (TWA) concentration. Another organic reagent used in the medical examination center, formaldehyde, did not exhibit co-adsorption along with the extraction of xylene by NTS. The use of a fume hood satisfactorily reduced VOC emissions in the workplace. Additionally, a management strategy involving chemical control banding was adopted to evaluate the emission control of xylene and formaldehyde in this work. We recommend that workers wear the NTS as a routine micro-sampler in order to protect their health.
\end{abstract}

Keywords: Exposure risk; Sampling; Solid phase microextraction; Xylene; Formaldehyde; Hospital.

\section{INTRODUCTION}

Indoor air quality depends on the activities of people, in particular, in some indoor work places, in which central air conditioning system, such as heating, ventilation, and air condition (HVAC) systems, influence indoor air quality (IAQ) (Rösch et al., 2014; Ayodele et al., 2016). Notably, the hospital is an almost completely indoor space with various medical activities continuously in progress. According to Wu et al.'s field survey at a teaching hospital in Taiwan, more hazardous chemicals and solvents are used in the medical examination center than in any other work rooms (Wu et al., 1998).

Xylene and formaldehyde are the main organic compounds that are used in a medical examination center (Fooyin Hospital, 2016). Human are exposed to xylene by inhalation, and the resulting symptoms include excitability, mental confusion, staggering, headache, dizziness, narcosis and coma. Formaldehyde mainly causes irritation of the eyes and respiratory tract, pulmonary edema, inflammation and pneumonia. Additionally, formaldehyde has been classified as a "probably carcinogenic agent" by the International Agency for Research on Cancer (IARC) (Kent, 1998). The

\footnotetext{
${ }^{*}$ Corresponding author.

Tel.: +886-7-781-1151 ext. 5113; Fax: +886-7-782-6735

E-mail address: PL031@fy.edu.tw
}

synergistic toxicology on humans for mixtures of xylene and formaldehyde has seldom been investigated. Many results of mouse tests have revealed that mixtures of xylene and formaldehyde can synergistically reduce mice memory, cause genetic damage in the liver, kidney and marrow, and increase the rate of sperm malformation (Bai, 2012; Wu et al., 2012).

Various sampling methods have been used to measure the emissions of multiple mixtures of indoor volatile organic compounds (VOCs). Can et al. (2015) measured VOC emissions, using $200 \pm 1 \mathrm{mg}$ 18-35 mesh granular activated carbon as the adsorbent, at a painting and printmaking department of an arts university in Turkey. Liang et al. (2014) used Tenax adsorption tubes with an active sampling flow rate of $200 \mathrm{~mL} \mathrm{~min}{ }^{-1}$, maintained for 10-20 min using a pump, in the interior of a construction sites of an apartment building in Beijing, China. Sax et al. (2006) and Loh et al. (2007) studied the indoor organic air pollutant emissions and health risks in the United States. Aromatic compounds and formaldehyde were typical of the compounds that were extracted using the site samplers. However, the co-adsorption of multiple compounds on the trap samplers has not been evaluated.

Solid-phase microextraction (SPME), used to sample gaseous organic VOCs, is environmentally friendly because it is solvent-free and can be reused (Lord et al., 2010). Cheng et al. (2011) sampled gaseous BTEXs using divinylbenzene (DVB) adsorbents packed in specially made SPME devices, called needle trap samplers (NTS). They successfully 
sampled emissions from indoor sources including mosquito coils, incense coil, essential oils, air fresheners, and paint emissions in the workplace (Cheng et al., 2013; Cheng and Lai, 2014; Cheng et al., 2014; Cheng et al., 2015; Cheng et al., 2016; Cheng et al., 2017). In the present work, NTS are used to measure personal exposure to VOCs during a medical examination at a teaching hospital. For comparison, VOC were actively sampled in the field simultaneously, following the approach of Özden Üzmez et al. (2015). The susceptible effect of co-adsorption for the multiple VOCs is evaluated. Finally, a management recommendation, based on the concept of chemical banding, is made.

\section{METHODS}

\section{Gaseous Compound Extraction Using Needle Trap Samplers}

The NTS extracts chemical compounds in air through needles by diffusion. Accordingly, a linear gaseous concentration profile $(C(Z)$ in Fig. 1$)$ is obtained along the diffusion path $(Z)$, and the extraction is characterized by the area $(A)$ of opening and the diffusion path length. The total amount $(n)$ of analyte extracted in a time interval $(t)$ is estimated as the following formula (Lord et al., 2010):

$$
n=D_{m} \frac{A}{Z} \int C(t) d t
$$

where $D_{\mathrm{m}}$ is the diffusion coefficient of a compound that is sampled by the sorbent in the needle. The quantity $(n)$ of the extracted analyte is assumed to be proportional to the total sample concentration over a time interval $(C(t))$ given a constant $D_{\mathrm{m}}$, a uniform needle opening $(A)$, and a fixed diffusion path distance $(Z)$.

\section{Preparation of Needle Trap Sampler}

The NTS comprised a stainless steel needle packed with DVB particles, mesh sizes 60-80. DVB particles were packed by aspiration until the desired length was $7 \mathrm{~mm}$ and then when the diffusion path was $3 \mathrm{~mm}$ long. A very small amount of epoxy glue was applied to the exposed portion of the sorbent layer to immobilize the sorbent particles. To prevent blockage of the NTS epoxy-resin plug, air was drawn continuously through the NTS packing phase as the epoxy cured. Finally, the DVB in an NTS was conditioned by heating at the injection port of the gas chromatography equipped with a flame ionization detector (GC-FID) under $260^{\circ} \mathrm{C}$ for $30 \mathrm{~min}$.
The uniformity of the packing phase in an NTS was examined by following the procedures to establish the desired sampling flow rate through an NTS $\left(\mathrm{mL} \mathrm{min}^{-1}\right)$ by drawing through the packing phase using an aspirating pump (Cheng et al., 2011; Cheng et al., 2013; Cheng and Lai, 2014; Cheng et al., 2014; Cheng et al., 2015; Cheng et al., 2016; Cheng et al., 2017). When the relative standard deviations (RSD) of the sampling flow rates across three duplicate tests did not exceed $5 \%$, the packed materials inside the NTS were assumed to be uniformly immobilized. BTEX standard gas samples (around $10 \mathrm{ppm}$ ) were prepared in a Pyrex glass bulb, in which the NTS was inserted for $1-2 \mathrm{~h}$ to extract BTEX. When the RSD of the extracted mass in triplicate tests were less than $5 \%$, the adsorption capacities in the NTS were assumed to be constant.

\section{Chemicals, Materials and Equipment}

Seven $22 \mathrm{G}$ stainless steel needles (length: $7 \mathrm{~cm}$; OD: $0.71 \mathrm{~mm}$; ID: $0.41 \mathrm{~mm}$ ) were purchased from a local company (Herling Co. Ltd., Pingtung, Taiwan) for preparing the NTS, in which DVB particles (Supelco, Bellefonte, PA, USA) were used as adsorbents. Aspirating pumps used to test the NTS sampling flow rates were purchased from Kitagawa (AP-20; Kawasaki, Japan), and epoxy glue was purchased from Nao-Pao Applied Material Co. Ltd. (Taoyuan, Taiwan). All gases (Jing-De Gas Co., Ltd., Kaohsiung, Taiwan) used in chromatographic analysis were of ultra-high purity.

Gaseous samples were analyzed using a GC (6890N; Agilent Technologies, Inc., Wilmington, DE, USA) that was equipped with an FID. The capillary column was HP19091Z-413 HP-1 PDMS $(30 \mathrm{~m} \times 320 \mu \mathrm{m} \times 0.25 \mu \mathrm{m})$ (Agilent Technologies, Inc.).

\section{Sampling and Analysis}

The target sampling site was the medical examination center of a teaching hospital in Donggang Township, Pingtung County, Taiwan. This general hospital was established at 1971, and the building has 11 flours. The main medical departments include Medicine, Surgery, Gynecology, Pediatrics, Emergency, Rehabilitation, Dermatology, Ophthalmology, and Non-clinical. The medical examiners collect samples from clinical patients for medical examination. Two highly hazardous solvents are used in medical examination: Xylene is used for slice staining, and formalin, which contains $40 \%$ formaldehyde, is used for fixation before slicing. Figs. 2(a) and 2(b) show the procedures that are followed by the technicians in the

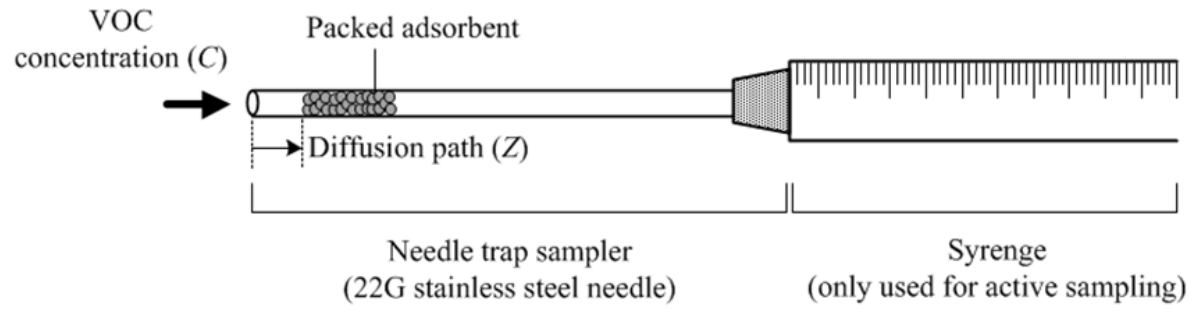

Fig. 1. Schematic needle trap sampler. 
(a)

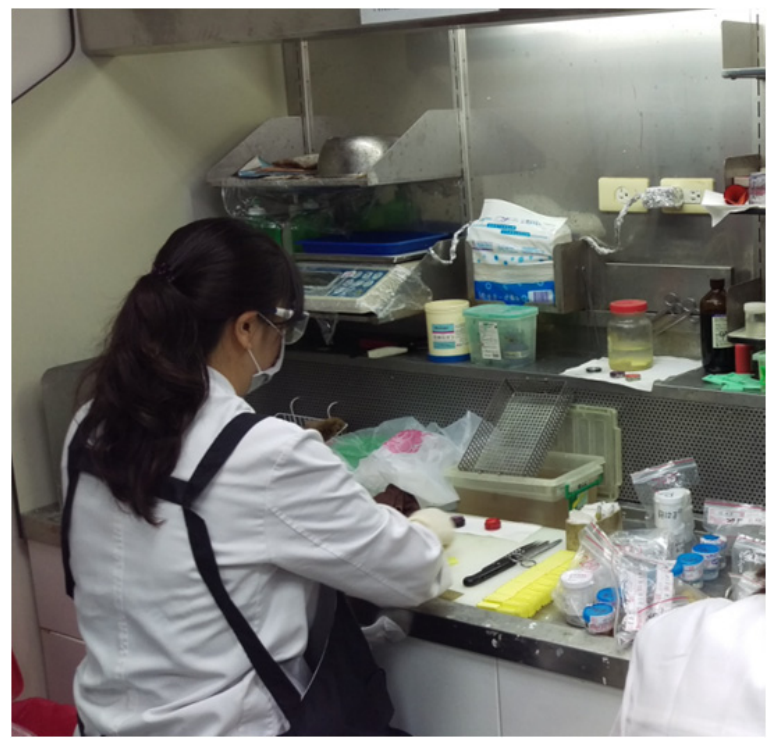

(b)

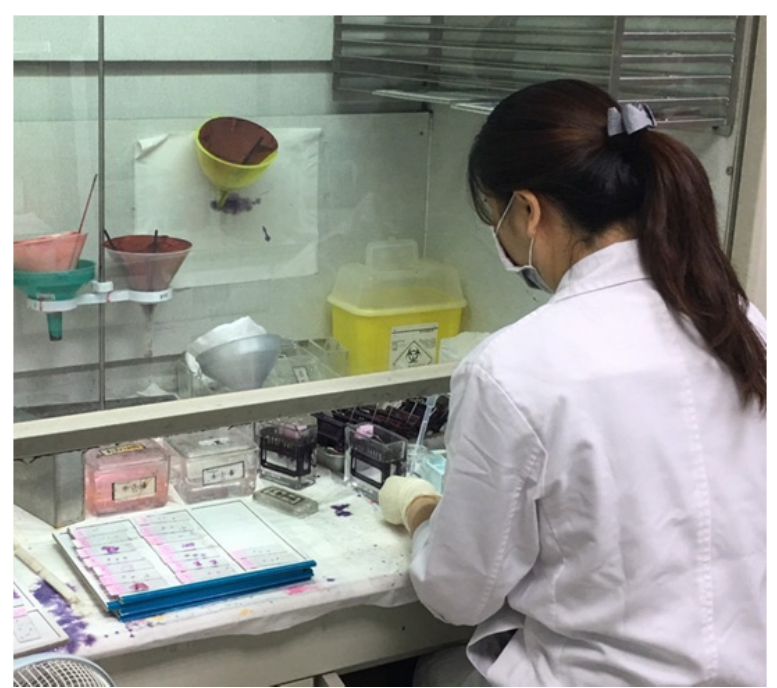

Fig. 2. Solvent use during the medical examination: (a) slice staining and (b) fixation of slice.

medical examination center. During the staining and fixation procedures, VOCs are emitted. Medical technicians use masks and gloves to prevent VOC exposure. Ventilation hoods are used to reduce indoor VOC emissions. To measure the workers' time-weighted average (TWA) exposure to VOCs, three samplers of the different types were worn simultaneously by medical technicians: (a) passive NTS sampling pens, developed by the authors' group; (b) detector tubes, manufactured by Gastec (Kanagawa, Japan); and (c) personal sampler that comprises a charcoal tube (Method 1501 for xylene), or an XAD adsorbent sampler (Method 2541 for formaldehyde) with a personal air pump for active sampling, which was developed by the U.S. National Institute for Occupational Safety and Health (2003). The NTS sampling pen (Fig. 3) is a microminiaturized sampler (with a mass of only $200 \mathrm{~g}$ and a length of $20 \mathrm{~cm}$ ), with auxiliary functions of detecting humidity and temperature. The stainless steel needle for extracting VOCs is installed inside the pen. (a)

(b)

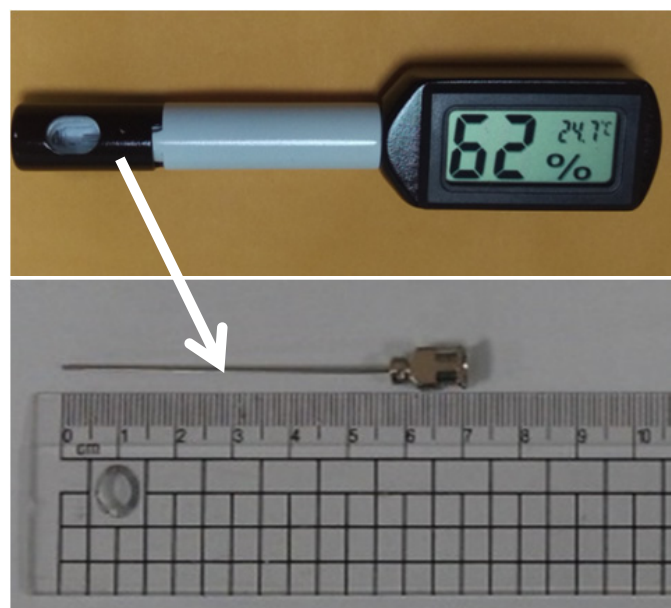

Fig. 3. The multiple functional NTS pens. (a) The appearance and its auxiliary functions, detectors of humidity and temperature. (b) The needle for extracting VOCs is inside the pen and its length is $7 \mathrm{~cm}$. The gas compounds diffuse into the side hole of sampling pen and then are extracted by the NTS.

After VOC sampling, an NTS was inserted into the injection ports of the GC-FID to desorb VOCs for analysis. The desorption time and temperature at the injection port were $30 \mathrm{~s}$ and $250^{\circ} \mathrm{C}$. The temperature of the $\mathrm{GC}$ was increased from $50^{\circ} \mathrm{C}$ in increments of $15^{\circ} \mathrm{C} \mathrm{min}{ }^{-1}$ to $180^{\circ} \mathrm{C}$ and then held for $2 \mathrm{~min}$. The FID detector was heated to $300^{\circ} \mathrm{C}$. The flow rate of the carrier gas, nitrogen, was $1.2 \mathrm{~mL} \mathrm{~min}^{-1}$, and the split-off operating mode was used. The following discussion section presents the calibration procedure and the way in which workers were exposed to the BTEX concentrations and the personal TWA concentrations of the mass that was extracted using NTS. The concentrations of xylene were obtained using the calibration formula in Table 1.

\section{RESULTS AND DISCUSSION}

\section{Determining Xylene Concentration and Confirming Co-Adsorption of Formaldehyde Using Needle Trap Samplers}

According to Eq. (1) (Lord et al., 2010), the total amount of extracted analyte $(n)$ is proportional to the interval of extraction $(t)$ and the concentration of VOC $(C)$, given that the coefficients $D_{\mathrm{m}}, Z$ and $A$ are fixed. Six

Table 1. The calibration equation of extracted mass of xylene and the corresponding integral analysis area by GC-FID.

\begin{tabular}{lll}
\hline VOCs & Calibration equations $^{\mathrm{a}}$ \\
\hline xylene & $y=2.6007 x-3.3366, R^{2}=0.9996$ & $(2)$ \\
\hline
\end{tabular}

Note:

${ }^{\mathrm{a}}$ Abbreviations in Eq. (2):

$y=$ the xylene concentration in ppm; $x=$ the integral area analyzed by GC-FID; and $R^{2}=$ the first order linear coefficient of regression. 
concentrations $(0,2,5,10,15$ and $20 \mathrm{ppm})$ of xylene were prepared in a Pyrex glass bulb with a volume of around $500 \mathrm{~mL}$. The NTS were inserted in series into the bulbs to conduct extraction tests for $1 \mathrm{~h}$. The extracted masses of xylene and the corresponding integrated areas of the peaks that were obtained by GC-FID are used to yield calibration equations in Table 1. The concentrations of xylene in the air samples in the medical examination center are determined using Eq. (2) and from the areas under the GC-FID peaks. If the practical sampling time with an NTS is $8 \mathrm{~h}(480 \mathrm{~min})$, then the measured concentrations of BTEXs must be multiplied by $0.125(60 \mathrm{~min} / 480 \mathrm{~min}=0.125)$ because the multiplying factor $(f)$ for time weighting is calculated as $f=60 \mathrm{~min} /$ (practical sampling time).

Earlier studies have thoroughly investigated the performance of NTS in the adsorptive extraction of aromatic compounds (Cheng et al., 2011; Cheng et al., 2013; Cheng and Lai, 2014; Cheng et al., 2014; Cheng et al., 2015; Cheng et al., 2016; Cheng et al., 2017). Formaldehyde is also emitted in the medical examination center, so an NTS extracted xylene and formaldehyde was tested. Experimental series of xylene $(10 \mathrm{ppm})$ and formaldehyde $(2,10$, and $20 \mathrm{ppm}$ ) were prepared in the Pyrex glass bulb, and then analyzed in duplicate using GC-FID to compare the areas under the peaks of xylene to each other. The mass of xylene that was extracted using an NTS was not significantly different in the presence of 2-20 ppm formaldehyde. In fact, mixing 10-ppm xylene with 2-20 ppm formaldehyde changed the extracted mass by less than $0.5 \%$. Several works have discussed the individual effects of humidity, temperature, BTEX matrices and atmospheric wind velocity on the SPME extraction of BTEX samplings, and these factors have been found to have no effects on the adsorption of BTEX on common commercial adsorbents such as polymethylsiloxane (PDMS), DVB and Carboxen (CAR) (Pawliszyn, 1997; Tsai and Kao, 2006; Schupfer and Huynh, 2008). However, this is the first to discuss the co-adsorption of formaldehyde and xylene by DVB adsorbent.

\section{Xylene Emissions in the Medical Examination Center}

Three methods were used to sample and examine the
TWA exposure of workers to xylene. A detector tube, a passive dositube (No. 122DL; GASTEC Corp.) was used to examine BTEX TWA concentrations. The detection limit for xylene was $2.13 \mathrm{ppm}$ with a sampling time of $8 \mathrm{~h}$. Five tests at the medical examination center revealed no color change so the workers were exposed to xylene TWA concentrations of less than $2.13 \mathrm{ppm}$.

Fig. 4 shows the TWA concentrations that were detected using passive NTS sampling pens and active charcoal tubes samplers (Method 1501). The differences between all xylene concentrations that were measured using the NTS and Method 1501 were in the range 4.2-6.0\%, indicating that the use of an NTS is an effective alternative to Method 1501 for the occupational monitoring of indoor gaseous aromatic compounds. However, the passive detector tube is not sufficiently sensitive for examining the exposure of workers to xylene.

Technicians were exposed to xylene concentrations of 0.11-1.2 ppm, which are far below the permissible exposure limit (PEL) 100 ppm (OSHA, 1992). The fume hood (Fig. 2(b)) satisfactorily protects the technician against emissions of VOCs while he or she fixes slices. The low crack height of the transparent sliding door of the fume hood is essential to keeping the capture speed high enough to reduce the exposure of workers to VOCs.

\section{Formaldehyde Emissions at the Medical Examination Center}

Formalin is used for fixation before slicing in medical examinations. Formalin emits gaseous formaldehyde, and a front side exhaust hood without sliding door (Fig. 2(a)) was used to protect technicians from those emissions. Two personal formaldehyde sampling methods, the use of a passive dositube (No. 91D, GASTEC Corp.) and active sampling Method 2541 (NIOSH, 2003), were simultaneously implemented. No color change occurred in the formaldehyde dositubes, indicating that the 8-h TWA exposure concentration was less than the detection limit 0.0625 ppm. Examination by GC-FID, followed by implementation of active sampling Method 2541, also yielded an 8-h TWA formaldehyde exposure concentration of less than the detection limit

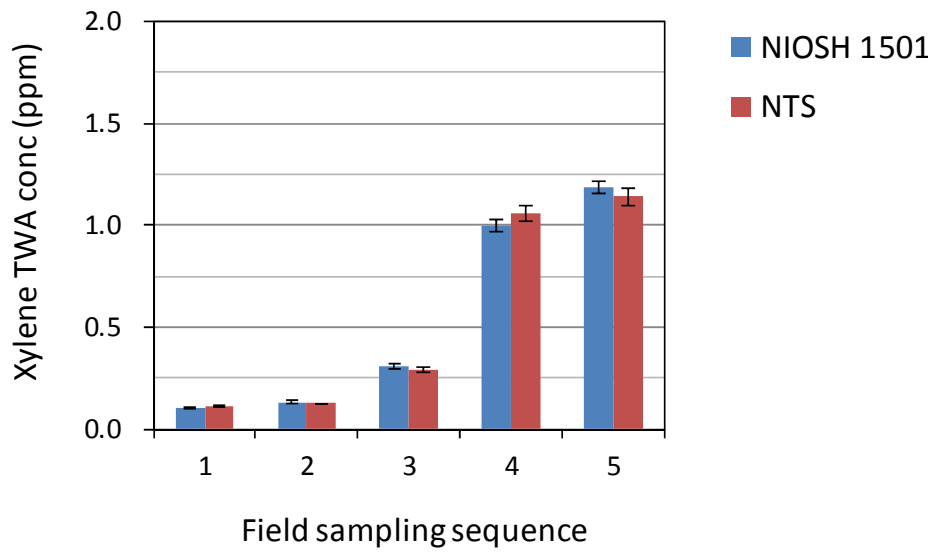

Fig. 4. The variations of emitted concentrations of xylene at different sampling sequences via personal sampling taken by passive needle trap samplers and active charcoal tubes. 
Table 2. The EHR of hazardous chemicals assessed in this study.

\begin{tabular}{lllll}
\hline Exposure Hazard Ranking ${ }^{\mathrm{a}, \mathrm{b}}(\mathrm{EHR})$ & Chemicals & PDC in sites $(\mathrm{ppm})$ & PEL $(\mathrm{ppm})$ & PDC/PEL $^{\mathrm{a}}(\%)$ \\
\hline 1 & Xylene & $0.11-1.2$ & 100 & $0.11-1.2$ \\
1 & Formaldehyde & $0.05-0.0625$ & 0.75 & $6.7-8.7$ \\
\hline
\end{tabular}

Note:

a PDC is "personal detection concentrations" and PEL is "permissible exposure limit". Rank 1: PDC/PEL < 50\%; Rank 2: PDC/PEL $\geq 50 \%$; and Rank 3: PDC/PEL $\geq 100 \%$.

${ }^{\mathrm{b}}$ The strategy of control for chemicals must be preserved as the currently existing approach for EHR Rank 1; and new strategies of control must be considered for EHR Rank 2 and 3 to improve the workers' exposure of chemicals.

$0.05 \mathrm{ppm}$. In summary, these field-analyzed concentrations of formaldehyde, 0.0625 or $0.05 \mathrm{ppm}$, are both lower than the PEL of 0.75 ppm (OSHA, 1992).

According to a field examination, the draught air speed $10 \mathrm{~cm}$ outside the exhaust hood was $2.2-3.0 \mathrm{~m} \mathrm{~s}^{-1}$ during the fixation procedures, meeting the operational standard (draught air speed of at least $2.0 \mathrm{~m} \mathrm{~s}^{-1}$ ) that was set by the Environmental and Occupational Health Center of the hospital.

\section{Assessing the Workers' Health Risk in the Medical Examination Center}

In Taiwan, the management of the health risks of workers who are exposed to hazardous chemicals is legislated using the concept of chemical control banding (Department of Labor, Taiwan, 2014). Typically, personal detection concentrations (PDCs) at work and the PELs of significant chemical compounds are used to calculate an Exposure Hazard Ranking (EHR). Table 2 demonstrates the EHRs for xylene and formaldehyde are Rank 1, meaning that the procedures for controlling chemical emissions at the medical examination center were highly successful, because the local ventilation systems are especially effective.

\section{CONCLUSIONS}

Based on this investigation, the NTS is recommended for use as a personal passive sampler in the medical examination center because it almost equals the sampling efficiency of Method 1501 of NIOSH (2003). In Taiwan, the Ministry of Labor requires harmful organic solvents and chemicals in the air of a working environment to be sampled and analyzed, followed by an official declaration that is submitted to the government, at least once every six months (Department of Labor, Taiwan, 2016). A multi-functional sampling pen with an NTS should be promoted as a sampler of organic compounds for routine monitoring purposes. Method 1501 requires workers to wear a sorbent tube connected to a pump in order to assess exposure to organic chemicals, but workers commonly complain about the inconvenience of carrying this equipment. Thus, this work evaluated the application of a mini-sampler in occupational health.

SPME sampling is environmentally friendly. Gałuszka et al. (2012) proposed that the objective of green analysis technology is to reduce hazardous substance use. Whereas the extraction of VOCs from activated carbon requires large amounts of organic solvents, sampling and analyzing pollutants via an NTS uses only small quantities of analytical reagents. Notably, the NTS can also be reused many times for sampling. As part of a management system that is based on chemical banding, the duplications in sampling and analyzing chemicals using NTSs in the workplace effectively control the risk associated with harmful chemicals.

\section{ACKNOWLEDGEMENTS}

The authors would like to thank the Fooyin University Hospital, Taiwan, for financially supporting this research under contract Nos. FH-HR-105-05 and FH-HR-106-04.

\section{REFERENCES}

Ayodele, C.O., Fakinle, B.S., Jimoda, L.A. and Sonibare, J.A. (2016). Investigation on the ambient air quality in a hospital environment. Cogent Environ. Sci. 2: 1215281.

Bai, H. (2012). Synergistic genotoxicity of formaldehyde and xylene for mice. The Shanxi Medical University, Master Dissertation, China.

Can, E., Üzmez, Ö.Ö., Döğeroğlu, T. and Gaga, E.O. (2015). Indoor air quality assessment in painting and printmaking department of a fine arts faculty building. Atmos. Pollut. Res. 6: 1035-1045.

Cheng, W.H., Zhan, W. and Pawliszyn, J. (2011). Extraction of gaseous VOCs using passive needle trap samplers. Aerosol Air Qual. Res. 11: 387-392.

Cheng, W.H., Zhan, W. and Pawliszyn, J. (2013). Gaseous and particle-bound VOC products of combustion extracted by needle trap samplers. J. Chin. Chem. Soc. 60: 1027-1032.

Cheng, W.H. and Lai, C.H. (2014). Sampling gaseous compounds from essential oils evaporation by solid phase microextraction devices. Atmos. Environ. 99: 124-129.

Cheng, W.H., Jiang, J.R., Lin. C., Liou, J.J., Wu, Z.H., Hsu, Y.H. and Yang, Z.Y. (2014). Preparation of needle trap samplers to extract air compounds from indoor electric-vaporizing sources. J. Air Waste Manage. Assoc. 64: 488-493.

Cheng, W.H., Lai, C.H., Tzeng, W.J., Her, C. and Hsu, Y.H. (2015). Gaseous products of incense coil combustion extracted by passive solid phase microextraction samplers. Atmosphere 6: 822-833.

Cheng, W.H., Tsai, D.Y., Lu, J.Y. and Lee, J.W. (2016). Extracting emissions from air fresheners using solid phase microextraction devices. Aerosol Air Qual. Res. 
16: 2362-2367.

Cheng, W.H., Huang, H.L, Chen, K.S. and Chang, Y.C. (2017). Quantification of VOC emissions from paint spraying on a construction site using solid phase microextraction devices. J. Environ. Sci. Health 52: $1158-1163$.

Department of Labor (2014). Act of hazardous chemical assessment and control banding (in Chinese), Taipei, Taiwan, Republic of China. http://laws.mol.gov.tw/FLAW/ FLAWDAT01.aspx?1sid=FL078716, Last Access: 26 March 2018.

Department of Labor (2016). Act of Environmental Monitoring in Working Place (in Chinese), Taipei, Taiwan, Republic of China. http://laws.mol.gov.tw/ FLAW/FLAWDAT01.aspx?1sid=FL015045, Last Access: 26 March 262018.

Fooyin University Hospital (2016). Investigation report on the chemicals used in a teaching hospital. Pingtung, Taiwan.

Gałuszka, A., Konieczka, P., Migaszewski, Z.M. and Namieśnik, J. (2012). Analytical Eco-Scale for assessing the greenness of analytical procedures. TrAC, Trends Anal. Chem. 37: 61-72.

Kent, C. (1998). Basics of toxicology. John Wiley and Sons, Inc., New York, NY.

Liang, W., Wang, C., Yang, C. and Yang, X. (2014). Volatile organic compounds in different interior construction stages of an apartment. Building Environ. 81: 380-387.

Loh, M.M., Levy, J.I., Spengler, J.D., Houseman, E.A. and Bennett, D.H. (2007). Ranking cancer risks of organic hazardous air pollutants in the United States. Environ. Health Perspect. 115: 1160-1168.

Lord, H., Zhan, W. and Pawliszyn, J. (2010). Fundamentals and applications of needle trap devices. Anal. Chim Acta 677: 3-18.

National Institute for Occupational Safety and Health. (2003). Manual of analytical methods. 4th Ed., Atlanta, GA, USA.

Occupational Safety and Health Administration (1992).
Permissible Exposure Limits (PELs) for Air Contaminants. Washington, DC, USA, http://www.dir.ca. gov/title8/ac1.pdf, Last Access: 26 March 26, 2018.

Özden, Ü.Ö., Gaga, E.O. and Döğeroğlu, T. (2015). Development and field validation of a new diffusive sampler for determination of atmospheric volatile organic compounds. Atmos. Environ. 107: 174-186.

Pawliszyn, J. (1997). Solid phase microextraction: Theory and practice. Wiley-VCH, Inc., New York, USA.

Rösch, C., Kohajda, T., Röder, S., von Bergen, M. and Schlink, U. (2014). Relationship between sources and patterns of VOCs in indoor air. Atmos. Pollut. Res. 5: 129-137.

Sax, S.N., Bennett, D.H., Chillrud, S.N., Ross, J., Kinney, P.L. and Spengler, J.D. (2006). A cancer risk assessment of inner-city teenagers living in New York City and Los Angeles. Environ. Health Perspect. 114: 1558-1566.

Schüpfer, P. and Huynh, C.K. (2008). Solid phase microextraction as a short-term sampling technique for BTEX occupational exposure. J. Occup. Environ. Hyg. 5: 490-500.

Tsai, S.W. and Kao, K.Y. (2006). Diffusive sampling of airborne furfural by solid-phase microextraction device with on-fiber derivatization. J. Chromatogr. A 1129: 2933.

Wu, P.C., Su, H.J., Huang, C.F., Lin, C.Y. and Lee C.C. (1998). Airborne microbial and chemical exposure assessment in a hospital environment. Chin. J. Pub. Health 17: 93-102.

Wu, Y.L., Chang, B.C. and Wu, S.H. (2012). The synergistic genotoxicity of aromatic compounds and formaldehyde from indoor decoration for mouse micronuclei and marrow. J Nanjing Xiaozhuang Univ. 6: 73-76.

Received for review, August 22, 2018 Revised, October 30, 2018 Accepted, November 19, 2018 